\title{
Infrared Bounds, Phase Transitions and Continuous Symmetry Breaking *
}

\author{
J. Fröhlich and B. Simon $\star \star$ \\ Department of Mathematics, Princeton University, Princeton, New Jersey 08540, USA
}

\section{T. Spencer}

Department of Mathematics, The Rockefeller University, New York, New York 10021, USA

\begin{abstract}
We present a new method for rigorously proving the existence of phase transitions. In particular, we prove that phase transitions occur in $(\boldsymbol{\phi} \cdot \boldsymbol{\phi})_{3}^{2}$ quantum field theories and classical, isotropic Heisenberg models in 3 or more dimensions. The central element of the proof is that for fixed ferromagnetic nearest neighbor coupling, the absolutely continuous part of the two point function in $k$ space is bounded by $0\left(k^{-2}\right)$. When applicable, our results can be fairly accurate numerically. For example, our lower bounds on the critical temperature in the three dimensional Ising (resp. classical Heisenberg) model agrees with that obtained by high temperature expansions to within $14 \%$ (resp. a factor of $9 \%$ ).
\end{abstract}

\section{§ 1. Introduction}

In this paper we develop a new method for establishing the existence of phase transitions or symmetry breaking for a class of ferromagnetic systems in $v \geqq 3$ dimensions. In particular we establish symmetry breaking for the classical isotropic Heisenberg model and the $(\boldsymbol{\phi} \cdot \boldsymbol{\phi})_{3}^{2}$ quantum field model. We also establish phase transitions for a broad class of lattice models having no internal symmetry. Work on this last issue has been done by Pirogov and Sinai [38] by very different methods.

It is well known $[34,35,25,3,6,2]$ that the $(\boldsymbol{\phi} \cdot \boldsymbol{\phi})^{2}$ model and, for finite range interactions, the isotropic Heisenberg model do not exhibit symmetry breaking in one or two dimensions. However for the case of long range interactions or in the presence of anisotropy phase transitions do occur in 2 or more dimensions $[15,40,1 \mathrm{~A}, 33,29]$.

To describe our strategy, let us consider the method of proof for the classical $v$-dimensional simple cubic Heisenberg model $[34,26]$. Let $F(\alpha-\beta)$ be the two point function $\left\langle\sigma_{\alpha} \cdot \sigma_{\beta}\right\rangle$ in the infinite volume periodic classical Heisenberg model

* Research supported by USNSF under grants GP-38048 and MPS-74-13252

$\star \star$ A. Sloan Fellow; also in the Department of Physics 
at zero magnetic field and at inverse temperature $J$, (we replace the usual $\beta J$ by $J$ ). Then we prove:

(A) The Fourier transform $\hat{F}(k)$ is of the form $[c \delta(k)+g(k)] d^{v} k$ where $0 \leqq g(k) \leqq$ $D J^{-1} k^{-2}$ for a universal constant $D$.

(B) A strictly positive lower bound $B$ on $F(0)$ as $J \rightarrow \infty$.

For the case at hand, step $B$ is trivial since $F(0)=\left\langle\sigma_{\alpha} \cdot \sigma_{\alpha}\right\rangle=1$. Step $A$ is proven in $\S 2$. The point is that when $v \geqq 3(\mathrm{~A})$ and (B) imply the existence of long range order, that is $F(\alpha) \nrightarrow 0=\left\langle\sigma_{\alpha}\right\rangle \cdot\left\langle\sigma_{\alpha}\right\rangle$ as $|\alpha| \rightarrow \infty$. The presence of long range order implies the existence of more than one ergodic component in the infinite volume theory. By proving (A) and (B) in non-zero field, one also establishes directly the existence of a non-zero spontaneous magnetization in many cases. For by (A) and (B) and the inequality $F(0)=(2 \pi)^{-v / 2} \int_{\left|k_{\imath}\right| \leqq \pi} \hat{F}(k)$,

$$
(2 \pi)^{v / 2} B \leqq c+D J^{-1} \int_{\left|k_{l}\right| \leqq \pi} k^{-2} d^{v} k .
$$

When $v \geqq 3$, the integral on the right of (1.1) converges, so for $J$ sufficiently large, $c$ is forced to be non-zero. Since, by (A) and the Riemann-Lebesgue lemma $c=(2 \pi)^{v / 2} \lim _{\alpha \rightarrow \infty} F(\alpha)$, there is long range order.

Our basic strategy thus emphasizes a kind of condensation at $k=0$ which should be thought of a spin-wave Bose condensation. It suggests that phase transitions are a very simple phenomenon in $v \geqq 3$ and only really subtle when $v=2$.

It is interesting that in the field theory case step $A$ is a consequence of the well known Källen-Lehmann representation

$$
F(k)=c \delta(k)+\int_{0}^{\infty} d \varrho(a)\left(p^{2}+a^{2}\right)^{-1}
$$

where $\int d \varrho(a)=1$. Step $(B)$ is the hard part of the proof.

We want to emphasize that the constant $D$ in (A) and (1.1) is independent of the single spin distribution and if one replaces $\left\langle\sigma_{\alpha} \cdot \sigma_{\beta}\right\rangle$ by $\left\langle\sigma_{\alpha}^{i} \cdot \sigma_{\beta}^{i}\right\rangle$, of the number of components. Our methods are also independent of the internal symmetry of the model. We can accomodate both models with no symmetry and those with continuous symmetry. On the other hand there are several restrictions on our results. We cannot handle general non cubic lattices and cannot directly deal with general non-nearest neighbor interactions (although using correlation inequalities [20A] one can often control this situation). Moreover, the extension to the quantum Heisenberg model is not immediate. [The extension has been accomplished by F. Dyson, E. Lieb and B. Simon (in prep.).]

In $\S 2$, we prove a general estimate of the form needed for step (A) and, in an appendix, we give an alternative proof. These estimates are a lattice analog of an estimate which one can obtain from the Källen-Lehmann representation and provide, via the convergence of the lattice approximation, a unified proof of these bounds in both the statistical mechanical and field theoretic cases.

In $\S 3$, we apply these bounds to prove phase transitions in certain lattice theories and in $\S 4$ to the field theory case. These bounds also have an application in handling difficult Peierls' type arguments [11].

It is a pleasure to thank F. Dyson, E. Lieb and O. McBryan for useful discussions. 


\section{§ 2. Gaussian Domination in Lattice Systems}

In this section, we will prove various replacements for the Källen-Lehmann representation (1.2) in lattice systems. Let $\Lambda$ be a subset of $\mathbb{Z}^{v}$ of the form of a rectilinear parallelpiped with $L_{1} \times \ldots \times L_{v}$ points. At each $\alpha \in \Lambda$, there is a random variable $\sigma_{\alpha} \in \mathbb{R}^{N}$ ( $N=$ fixed integer). We define the Hamiltonians in a periodic box $\Lambda$

$$
H_{\Lambda}(\{\sigma\})=-J \sum_{|\alpha-\beta|=1} \sigma_{\alpha} \cdot \sigma_{\beta}
$$

where the sum includes each pair of nearest neighbors once including points at opposite ends of $\Lambda$ which are nearest neighbors when $\Lambda$ is viewed as a torus. (Magnetic fields can be put into the single spin distributions below.) One chooses a measure $d \lambda$ on $\mathbb{R}^{N}$ with $\int \exp \left(a \sigma^{2}\right) d \lambda<\infty$ for all $a$; e.g. for the classical Heisenberg model in a field $\boldsymbol{\mu}: d \lambda=\exp (\boldsymbol{\mu} \cdot \boldsymbol{\sigma}) \delta(|\boldsymbol{\sigma}|-1) d^{3} \sigma$. In the standard way [41], we define a measure $d \mu_{\Lambda}$ on $\mathbb{R}^{N|\Lambda|}$ by

$$
d \mu_{\Lambda}=Z_{\Lambda}^{-1} \exp \left(-H_{\Lambda}(\{\sigma\})\right) \prod_{\alpha \in \Lambda} d \lambda\left(\sigma_{\alpha}\right)
$$

where $Z_{\Lambda}^{-1}$ is a constant chosen so that $d \mu_{A}$ is a probability measure. $\langle 0\rangle_{\Lambda}$ will denote expectation with respect to $d \mu_{\Lambda}$.

Given a function $h$ with values in $\mathbb{R}^{N}$ we define $\sigma(h)$ by $\sigma(h)=\sum h(\alpha) \cdot \sigma_{\alpha}$ and

$$
\begin{aligned}
\left(\partial_{i} h\right)(\alpha) & =h\left(\alpha+\delta_{i}\right)-h(\alpha) \\
\left(\partial_{i}^{*} h\right)(\alpha) & =h\left(\alpha-\delta_{i}\right)-h(\alpha) \\
(-\Delta h)(\alpha) & =2 v h(\alpha)-\sum_{i=1}^{v}\left[h\left(\alpha+\delta_{i}\right)+h\left(\alpha-\delta_{i}\right)\right]
\end{aligned}
$$

where $\delta_{i}$ is the vector $\left(\delta_{i}\right)_{j}=\delta_{i j}$ and $\alpha+\delta_{i}$ is defined $\bmod L_{i}$. Notice that

$$
-\Delta=\sum_{i=1}^{v} \partial_{i}^{*} \partial_{i}=\sum_{i=1}^{v} \partial_{i} \partial_{i}^{*}
$$

We propose to prove:

Theorem 2.1. For any $h_{1}, \ldots, h_{v}$ with values in $\mathbb{R}^{N}$ :

$$
\left\langle\exp \left[\sigma\left(\sum_{1}^{v} \partial_{i} h_{i}\right)\right]\right\rangle_{\Lambda} \leqq \exp \left[(2 J)^{-1} \sum_{\alpha, i}\left(h_{i}(\alpha)\right)^{2}\right] .
$$

Theorem 2.2. Under the hypotheses of Theorem 2.1:

$$
\left\langle\left[\sigma\left(\sum_{1}^{v} \partial_{i} h_{i}\right)\right]^{2}\right\rangle_{1} \leqq J^{-1} \sum_{x, i}|h(\alpha)|^{2}
$$

Theorem 2.3. For any $h$ with values in $\mathbb{R}^{N}$ :

$$
\langle\sigma(h) \sigma(-\Delta h)\rangle_{\Lambda} \leqq J^{-1} \sum_{\alpha, i}|h(\alpha)|^{2} .
$$

Remarks. 1. (2.1) is motivated by the so called grad $\phi$ bounds of canonical quantum field theory first proven by Glimm and Jaffe [17] in their exponential form $[20,24]$. (See Herbst [24] for a general version of these bounds.) If one uses the 
convergence of the lattice approximation to field theories $[23,36],(2.1)$ implies these grad $\phi$ bounds.

2. (2.3) is the inequality we need in our proof of phase transitions below. It is better than the result announced in [13] by a factor of $2 v$ leading to improvements of a factor of 6 in the "transition temperatures quoted there: The constants in (2.1)-(2.3) are all best possible since the inequalities can be made arbitrarily close to equalities by taking $d \lambda$ close to a suitable Gaussian. The right side of the inequality is the worst possible Gaussian case, hence the name Gaussian domination.

3. (2.1)-(2.3) carry over to the infinite volume limit of periodic states.

4. We give an alternative proof of (2.3) with a worse constant in the appendix.

Proof of Theorem 2.2 given (2.1)

Since $\langle\cdot\rangle$ is translation invariant, and $\sum_{\alpha}\left(\partial_{i} h_{i}\right)(\alpha)=0,\left\langle\sigma\left(\partial_{i} h_{i}\right)\right\rangle=0$. Thus

$$
\begin{aligned}
& \lim _{\lambda \rightarrow 0}\left\{\left\langle\exp \left(\lambda \sigma\left(\sum_{1}^{v} \partial_{i} h_{i}\right)\right)\right\rangle-1\right] \lambda^{-2}=\frac{1}{2}\left\langle\sigma\left(\sum \partial_{i} h_{i}\right)^{2}\right\rangle . \\
& \left.\lim _{\lambda \rightarrow 0} \exp \left[(2 J)^{-1} \lambda^{2} \sum_{\alpha, i}\left|h_{i}(\alpha)\right|^{2}\right]-1\right\} \lambda^{-2}=(2 J)^{-1} \sum_{\alpha}\left|h_{i}(\alpha)\right|^{2}
\end{aligned}
$$

(2.1) implies (2.2).

\section{Proof of Theorem 2.3 given (2.2)}

View the functions on $\Lambda$ with values in $\mathbb{R}^{N}$ as vectors in a Hilbert space in the natural way. Define linear operators $A_{i}$ by

$$
\begin{aligned}
A_{i} f & =\partial_{i}^{*}(-\Delta)^{-1 / 2} f, \quad f \in \operatorname{Ran}(-\Delta) \\
& =0, \quad f \in \operatorname{Ker}(-\Delta)=\text { constants } .
\end{aligned}
$$

We first note it suffices to prove (2.3) for $h \in \operatorname{Ker}(-\Delta)^{\perp}$, since letting $\tilde{h}=h-c \in$ $\operatorname{Ker}(-\Delta)^{\perp}, \sum_{\alpha}|h(\alpha)|^{2} \leqq \sum_{\alpha}|h(\alpha)|^{2}$ and $\langle\sigma(h) \sigma(-\Delta h)\rangle=\langle\sigma(\tilde{h}) \sigma(-\Delta \tilde{h})\rangle$ because $\Delta h=\Delta \tilde{h}$ and $\langle\sigma(-\Delta h)\rangle=0$ by translation invariance.

$$
\text { Let } h_{i}=A_{i} h \text {. Then } \sum_{\alpha, i}\left|h_{i}(\alpha)\right|^{2}=\sum_{i}\left\langle h_{i}, h_{i}\right\rangle=\left\langle h, \sum A_{i}^{*} A_{i} h\right\rangle=\langle h, h\rangle \text { since } \sum A_{i}^{*} A_{i}=1
$$
on $(\operatorname{Ker}-\Delta)^{\perp}$. Moreover, $\sum_{1}^{v} \partial_{i} h_{i}=(-\Delta)(-\Delta)^{-1 / 2} h=(-\Delta)^{1 / 2} h$, so

$$
\begin{aligned}
\left\langle\left[\sigma\left(\sum_{1}^{v} \partial_{i} h_{i}\right)\right]^{2}\right\rangle & =\sum_{\alpha, \beta}\left\langle\sigma_{\alpha} \sigma_{\beta}\right\rangle\left(-\Delta^{1 / 2} h\right)(\alpha)\left(-\Delta^{1 / 2} h\right)(\beta) \\
& =\sum_{\alpha, \beta}\left\langle\sigma_{\alpha} \sigma_{\beta}\right\rangle(-\Delta h)(\alpha) h(\beta) \\
& =\langle\sigma(h) \sigma(-\Delta h)\rangle
\end{aligned}
$$

where the second step follows by noting that since $\left\langle\sigma_{\alpha} \sigma_{\beta}\right\rangle$ is only a function of $\alpha-\beta$ it is the kernel of a operator on $l^{2}$ commuting with $-\Delta$ and so with $(-\Delta)^{1 / 2}$.

The above reduces everything to the proof of (2.1). As a preliminary to this proof, we rewrite (2.1) in a more suggestive form. First, by a "summation by parts" 
$\sigma\left(\partial_{i} h_{i}\right)=\sum_{\alpha} \sigma_{\alpha}\left(h_{i}\left(\alpha+\delta_{i}\right)-h_{i}(\alpha)\right)=\sum_{\alpha} h_{i}(\alpha)\left(\sigma_{\alpha-\delta_{2}}-\sigma_{\alpha}\right)$. Thus (2.1) can be written:

$$
\left\langle\exp \left[\sum_{|\alpha-\beta|=1} h_{\alpha, \beta}\left(\sigma_{\alpha}-\sigma_{\beta}\right)\right]\right\rangle \leqq \exp \left((2 J)^{-1} \sum_{|\alpha-\beta|=1}\left|h_{\alpha \beta}\right|^{2}\right)
$$

where there is a "direction" given to each "bond", $(\alpha, \beta)$ with $|\alpha-\beta|=1$. Now define $d \tilde{\lambda}=\exp \left(\frac{1}{2} J \sigma^{2}\right) d \lambda$ and

$$
Z\left(h_{\alpha \beta}\right)=\int \prod_{\alpha} d \tilde{\lambda}\left(\sigma_{\alpha}\right) \exp \left[\underset{|\alpha-\beta|=1}{-} \frac{1}{2} J\left(\sigma_{\alpha}-\sigma_{\beta}-J^{-1} h_{\alpha \beta}\right)^{2}\right] .
$$

Then (2.1) is equivalent to:

$$
Z\left(h_{\alpha \beta}\right) \leqq Z(0)
$$

for all $h_{\alpha \beta}$.

The key to the proof is the elementary

Lemma 2.4. Let $\mu, v$ be (not necessarily positive) measures on $\mathbb{R}^{M}$. Then, for any $h \in \mathbb{R}^{M}$

$$
\left|\int d \mu(\sigma) d v\left(\sigma^{\prime}\right) f\left(\sigma-\sigma^{\prime}-h\right)\right| \leqq\left[\int d \mu(\sigma) f\left(\sigma-\sigma^{\prime}\right) d \mu\left(\sigma^{\prime}\right)\right]^{1 / 2}\left[\int d v(\sigma) f\left(\sigma-\sigma^{\prime}\right) d v\left(\sigma^{\prime}\right)\right]^{1 / 2}
$$

for any positive definite function $f$ and, in particular, for $f(x)=\exp \left(-\frac{1}{2} J x^{2}\right)$.

Proof. Let $(\mu, v) \equiv \int d \mu(\sigma) d v\left(\sigma^{\prime}\right) f\left(\sigma-\sigma^{\prime}\right)$. Let $v_{h}(\cdot)=v(\cdot-h)$. Then

$$
\left|\left(\mu, v_{h}\right)\right| \leqq(\mu, \mu)^{1 / 2}\left(v_{h}, v_{h}\right)^{1 / 2}=(\mu, \mu)^{1 / 2}(v, v)^{1 / 2}
$$

which proves the lemma.

Proof of Theorem 2.1. Suppose first that $d \tilde{\lambda}(\sigma)=F(\sigma) d \sigma$. We use a transfer matrix formalism. Write an index $\alpha \in \Lambda$ by $\alpha=(i, \beta), i=1, \ldots, L_{1} ; \beta \in \Lambda^{(1)} \equiv\left(1, L_{2}\right) \times \ldots \times$ $\left(1, L_{v}\right)$. Define

$$
F_{i}\left(\left\{\sigma_{\beta}\right\}\right)=\left[\prod_{\beta \in \Lambda^{(1)}} F\left(\sigma_{\beta}\right)\right] \exp \left[-\sum_{\left(\beta-\beta^{\prime}\right)=1} \frac{1}{2} J\left(\sigma_{\beta}-\sigma_{\beta^{\prime}}-h_{\beta \beta^{\prime}}^{(i)}\right)^{2}\right]
$$

where $h_{\beta \beta^{\prime}}^{(i)}=h_{(i, \beta),\left(i, \beta^{\prime}\right)}$. We view $F_{i}$ as a multiplication operator on $L^{2}\left(\mathbb{R}^{M}\right)$ with $M=N L_{2} \ldots L_{v}$. For $\boldsymbol{a}$ in $\mathbb{R}^{M}$, let $T_{a}$ be the integral operator on $L^{2}\left(\mathbb{R}^{M}\right)$ with kernel $\exp \left(-\frac{1}{2} J\left(\sigma-\sigma^{\prime}-a\right)^{2}\right)$. Let $a_{i}$ be the vector with components $\left(a_{i}\right)_{\beta}=h_{(i, \beta)(i+1, \beta)}$. Then

$$
Z\left(\left\{h_{\alpha \beta}\right\}\right)=\operatorname{Tr}\left(\prod_{i=1}^{L_{1}} F_{i} T_{a_{2}}\right) .
$$

Lemma 2.4 can be rephrased:

$$
\left(f, T_{a} g\right) \leqq\left(f, T_{0} f\right)^{1 / 2}\left(g, T_{0} g\right)^{1 / 2}
$$

which implies that

$$
\left\|T_{0}^{-1 / 2} T_{a} T_{0}^{-1 / 2}\right\| \leqq 1 .
$$

By the cyclicity of the trace

$$
\operatorname{Tr}\left(\prod_{i=1}^{L_{1}} F_{i} T_{a_{i}}\right)=\operatorname{Tr}\left(\prod_{i=1}^{L_{1}}\left(T_{0}^{1 / 2} F_{i} T_{0}^{1 / 2}\right)\left(T_{0}^{-1 / 2} T_{a_{i}} T_{0}^{-1 / 2}\right)\right)
$$


so by $\left(2.4^{\prime}\right),(2.6)$ and Hölder's inequality [39] in the form

$$
\operatorname{Tr}\left(\prod_{i=1}^{N} A_{i} B_{i}\right) \leqq \prod_{i=1}^{N} \operatorname{Tr}\left(\left|A_{i}\right|^{N}\right)^{1 / N} \prod_{i=1}^{N}\left\|B_{i}\right\|
$$

we have

$$
Z\left(\left\{h_{\alpha \beta}\right\}\right) \leqq \prod_{i=1}^{L_{1}} \operatorname{Tr}\left(\left(F_{i} T_{0}\right)^{L_{1}}\right)^{1 / L_{1}} .
$$

Defining $h_{\alpha \beta}^{(i)}$ suitably with the property that $h_{\alpha \beta}^{(i)}=0$ if $\beta=\alpha+\delta_{1}$, (2.7) can be rewritten

$$
Z\left(\left\{h_{\alpha \beta}\right\}\right) \leqq \prod_{i=1}^{L_{1}} Z\left(\left\{h_{\alpha \beta}^{(i)}\right\}\right)^{1 / L_{1}} .
$$

Repeating this in the other directions (2.1) results. (2.1) for the special case $d \tilde{\lambda}=$ $F(\sigma) d \sigma$ implies (2.1) for all $d \lambda$ by a limiting argument.

(2.1)-(2.3) have some interesting consequences:

Corollary 2.5. Suppose that $v \geqq 3$. Let \langle\rangle be an infinite volume limit of \langle\rangle$_{A}$ 's where we may also vary the d $\lambda$ 's after taking $|\Lambda|$ to $\infty$. Suppose that \langle\rangle is an ergodic state ("pure phase"). Let $\hat{\sigma}_{\alpha}=\sigma_{\alpha}-\left\langle\sigma_{\alpha}\right\rangle$ and let $h$ be a map from $\mathbb{Z}^{v}$ to $\mathbb{R}^{N}$. Then:

$$
\left\langle e^{\hat{\sigma}(h)}\right\rangle \leqq \exp \left((2 J)^{-1}\left(h,(-\Delta)^{-1} h\right)\right)
$$

for any $h$ of compact support. In particular

$$
\left\langle\sigma(h)^{2}\right\rangle-\langle\sigma(h)\rangle^{2} \leqq J^{-1}\left(h,(-\Delta)^{-1} h\right) .
$$

Proof. First suppose that we are in finite volume and $\sum h(\alpha)=0$. Letting $h_{i}=\Delta^{-1} \partial_{i} h$ so that $\hat{\sigma}\left(\sum \partial_{i} h_{i}\right)=\sigma(h)=\hat{\sigma}(h)$ we see by $(2.1)$ that

$$
\left\langle e^{\hat{\sigma(h)}}\right\rangle_{\Lambda} \leqq \exp \left((2 J)^{-1}\left(h,(-\Delta)^{-1} h\right)\right)
$$

where $(-\Delta)^{-1}$ is $\Lambda$ dependent. Taking $\Lambda \rightarrow \infty$ on both sides we obtain (2.8) for $h$ with $\sum h(\alpha)=0$. For arbitrary $h$ of compact support, let $h_{\Lambda}=h-|\Lambda|^{-1}\left(\sum h\right) \chi_{\Lambda}$; for $\Lambda$ a cube. Since $\left(\Delta^{-1}\right)_{i j} \sim|i-j|^{2-v}$ at infinity $\left\langle\chi_{\Lambda},(-\Delta)^{-1} \chi_{\Lambda}\right\rangle \sim|\Lambda|^{1+2 v^{-1}}$ as $|\Lambda| \rightarrow \infty$. Thus $\left(h_{A},(-\Delta)^{-1} h_{A}\right) \rightarrow\left(h,(-\Delta)^{-1} h\right)$. Moreover, by ergodicity $\left\langle\hat{\sigma}\left(h_{A}\right)^{n}\right\rangle \rightarrow$ $\left\langle\hat{\sigma}(h)^{n}\right\rangle$ as $|\Lambda| \rightarrow \infty$. Thus (2.8) extends to all $h$. (2.9) follows from (2.8) as (2.2) followed from (2.1).

Caution. Alas (2.9) does not imply $\left\langle\sigma_{\alpha} \sigma_{\beta}\right\rangle-\left\langle\sigma_{\alpha}\right\rangle\left\langle\sigma_{\beta}\right\rangle$ is pointwise bounded by $(-\Delta)_{\alpha \beta}^{-1}$.

(2.9) implies a bound on a critical exponent:

Theorem 2.6. If $\left\langle\sigma_{\alpha} \sigma_{\beta}\right\rangle_{T} \sim|\alpha-\beta|^{-v+2-\eta}$, as $|\alpha-\beta| \rightarrow \infty$, for some infinite volume (critical) ergodic state, then for $v \geqq 3$,

$$
\eta \geqq 0 \text {. }
$$

Remark. For the field theory case this is a remark of Glimm-Jaffe [18]. 
Proof. Under the hypothesis $\left\langle\sigma_{\alpha} \sigma_{\beta}\right\rangle_{T} \geqq C(|\alpha-\beta|+1)^{-\eta^{\prime}}$ one sees that

$$
\left\langle\sigma\left(\chi_{\Lambda}\right)^{2}\right\rangle_{T} \geqq|\Lambda|^{2-\eta^{\prime} v^{-1}} \text {. }
$$

As noted above $\left\langle\chi_{A},(-\Delta)^{-1} \chi_{\Lambda}\right\rangle \sim|\Lambda|^{1+2 v^{-1}}$. Thus $1+2 v^{-1} \geqq 2-\eta^{\prime} v^{-1}$ or $\eta^{\prime} \geqq v-2$.

\section{§ 3. Phase Transitions in Classical Lattice Systems}

Consider the simplest lattice system of spins with values in $S^{N+1}$ on a $v$-dimensional lattice:

Theorem 3.1. For $N$-component "spherical" spins on a v-dimensional lattice ( $v \geqq 3)$ with nearest neighbor coupling, $J=T^{-1}$ the long-range order parameter

$$
c=\lim _{\alpha \rightarrow \infty} \sum_{j=1}^{N}\left\langle\sigma_{\alpha}^{(j)} \sigma_{0}^{(j)}\right\rangle
$$

obeys:

$$
c \geqq\left(1-\frac{1}{2} N I(v) T\right)
$$

where

$$
I(v)=(2 \pi)^{-v} \int_{\left|k_{i}\right| \leqq \pi}\left(\sum_{i=1}^{v} 1-\cos k_{i}\right)^{-1} d^{v} k .
$$

In particular, $c \neq 0$ if $T \leqq \tilde{T}_{c}$, where

$$
\tilde{T}_{c}=\left(\frac{1}{2} N I(v)\right)^{-1} .
$$

Proof. Let $F_{j}(\alpha)=\left\langle\sigma_{\alpha}^{(j)} \sigma_{0}^{(j)}\right\rangle$ for some fixed $j$. Define $d \omega_{j}$ by:

$$
F_{j}(\alpha)=\int_{\left|k_{i}\right| \leqq \pi} e^{i \alpha k} d \omega_{j}(k)
$$

$d \omega_{j}$ is a positive measure, since $F$ is positive definite. By (2.3)

$$
2(2 \pi)^{v} \int \sum_{i=1}^{v}\left(1-\cos k_{i}\right)|\hat{h}(k)|^{2} d \omega_{j}(k) \leqq\left(J^{-1}\right) \int|\hat{h}(k)|^{2} d^{v} k
$$

where

$$
\hat{h}(k)=(2 \pi)^{-v / 2} \sum_{\alpha} e^{-i k \alpha} h(\alpha)
$$

is normalized so that $\int|\hat{h}(k)|^{2} d^{2} k=\sum_{\alpha}|h(\alpha)|^{2}$. Peaking $\hat{h}$ near some $k_{0}$ we see that

$$
d \omega_{j}(k)=\left[D_{j} \delta(k)+g_{j}(k)\right] d^{v} k
$$

with

$$
\sum_{i=1}^{v}\left(1-\cos k_{i}\right)\left(g_{j}(k)\right) \leqq(2 \pi)^{-v}(2 J)^{-1} .
$$


Thus summing over $j$

$$
\begin{aligned}
1=\sum_{j=1}^{N}\left\langle\sigma_{0}^{(j)} \sigma_{0}^{(j)}\right\rangle & =c+\sum_{j=1}^{N} \int g_{j}(k) d^{v} k \\
& \leqq c+\frac{1}{2} N I(v) . \quad \square
\end{aligned}
$$

Dyson has pointed out to us that $I(3)$ has been exactly computed by Watson [44] (see also [28]) in terms of elliptic integrals: $I(3)=0.5054620197 \ldots$ so that we have for the classical Heisenberg and Ising models for $v=3$ :

$$
\begin{aligned}
& \tilde{T}_{c}(N=3, v=3)=1.31893 \\
& \tilde{T}_{c}(N=3, v=3)=3.95678
\end{aligned}
$$

to be compared with the values obtained from high temperature series (not rigorous but believed to be close to exact) [4]:

$$
\begin{array}{ll}
T_{c}(N=3, v=3)=1.44 \pm 0.02 & \left(=\tilde{T}_{c} \times(1.09)\right) \\
T_{c}(N=1, v=3)=4.51080 & \left(=\tilde{T}_{c} \times(1.14)\right) .
\end{array}
$$

Our accuracy for the Ising model is comparable to that obtained with the most sophisticated Peierls type argument combining self-avoiding random walk ideas with a contour type expansion [14].

Equation (3.3) has a useful interpretation. If one considers models with $N$ components and $\sum \sigma_{i}^{2}=N$, (3.3) implies a transition temperature $T_{c}^{\prime}(N)>T_{\infty}=$ $2 I(v)^{-1} \cdot T_{\infty}$ is easily seen to be the exact transition temperature in the spherical model [26] which is the $N \rightarrow \infty$ limit of this family of models [26,44]. Thus, we expect that (3.3) is exact to order $N^{-1}$ as $N \rightarrow \infty$.

We also note that in case one has a Lee-Yang theorem (at this point for $n=1$ [30], 2 or 3 [5]), the lower bound on $c^{1 / 2}$ is also a lower bound on the spontaneous magnetization, which, in particular goes to its $T=0$ value of 1 as $T \rightarrow 0$. For, since $d \lambda$ does not enter in our arguments, (3.1) continues to hold in non-zero field. But, by Lee-Yang, $\left\langle\sigma_{\alpha} \sigma_{\beta}\right\rangle$ clusters if $\boldsymbol{h} \neq 0$ so $c=\sum_{i=1}^{n}\left\langle\sigma_{0}^{(i)}\right\rangle^{2}$. But if $\boldsymbol{h}$ is in the 1-direction, $\left\langle\sigma_{0}^{i}\right\rangle=0$ if $i \neq 1$.

For the general nearest neighbor model, part (B) of our method is not much harder than the rather trivial considerations in Theorem 3.1:

Lemma 3.2. Let \langle\rangle$_{J, \lambda}$ denote the infinite volume expectation (may be dependent on choice of subsequences) for a lattice model with apriori finite measure $d \lambda$ on $\mathbb{R}^{N}$ and coupling $J$ between nearest neighbors. Let

$$
m=\max \{|\sigma| \mid \sigma \in \operatorname{supp} \lambda\}
$$

( $m$ may be infinite). Then

$$
\lim _{J \rightarrow \infty}\left\langle\left|\sigma_{0}\right|^{2}\right\rangle=m^{2} \text {. }
$$

Proof. Let $Z(J, \Lambda)$ be the partition function for finite volume. Then if $m$ is finite:

$$
Z(J, \lambda) \leqq\left|\lambda\left(\mathbb{R}^{N}\right)\right|^{|\Lambda|} e^{2 v J m^{2}|\Lambda|} .
$$


For any $m_{1}<m$, we can find a set $S_{m_{1}} \subset \mathbb{R}^{N}$ so that $\lambda\left(S_{m_{1}}\right)>0$ and $\sigma \cdot \sigma^{\prime} \geqq\left(m_{1}\right)^{2}$ for all $\sigma, \sigma^{\prime} \in S_{m_{1}}$. Thus:

$$
Z(J, \Lambda) \geqq\left|\lambda\left(S_{m_{1}}\right)\right|^{|\Lambda|} e^{2 v J m_{1}^{2}|\Lambda|} .
$$

From (3.7) and (3.8) we obtain a bound on the pressure

$$
\lim _{J \rightarrow \infty} P(J) / J=2 v m^{2},
$$

(since $m_{1}<m$ is arbitrary). (3.9) easily implies that

$$
\lim _{J \rightarrow \infty}\left\langle\sum_{|\beta|=1} \sum_{i=1}^{N} \sigma_{0}^{(i)} \sigma_{\beta}^{(i)}\right\rangle_{J}=2 v m^{2},
$$

since $P$ is convex and the expectation on the left of (3.10) lies between the left and right derivative of $P$ at $J$. But by the Schwarz inequality

$$
\left\langle\sigma_{0}^{(i)} \sigma_{\beta}^{(i)}\right\rangle \leqq\left\langle\left(\sigma_{0}^{(i)}\right)^{2}\right\rangle
$$

so that (3.10) and the obvious fact $\left\langle\left|\sigma_{0}\right|^{2}\right\rangle \leqq m^{2}$ lead to (3.6).

Theorem 3.3. In three or more dimensions, any lattice gas with an apriori measure invariant under $\sigma \rightarrow-\sigma$ has a phase transition for large enough $J$ in the sense that the periodic state is not ergodic.

Proof. Given Lemma 3.2, the same as the proof of Theorem 3.1.

Remarks. 1. The long range order parameter $c$ clearly approaches the "zero temperature value", $m^{2}$.

2. For one component spins, in two or more dimensions, a result of this genre has recently been proven by van Beijeren and Sylvester [1].

Actually symmetry doesn't play an especially important role in our method of proving phase transitions. We first prove a technical lemma.

Lemma 3.4. Fix a measure $d \lambda$ on $\mathbb{R}$ with $\int e^{a \sigma^{2}} d \lambda<\infty$ for all a, so that $\operatorname{supp} \mu$ intersects both $(-\infty, 0)$ and $(0, \infty)$. Let

$$
P(J, \mu)=\lim _{|\Lambda| \rightarrow \infty}|\Lambda|^{-1} \ln \left[\int \prod_{i \in \Lambda} d \lambda\left(\sigma_{i}\right) \exp \left(\mu \sum \sigma_{i}+J \sum_{|i-j|=1} \sigma_{i} \sigma_{j}\right)\right] .
$$

Then for suitable constants $\varepsilon$ and $\delta>0$

$$
P(J, \mu)-P(0, \mu) \geqq \varepsilon J-\delta
$$

for all $J \geqq 0, \mu$.

Proof. Suppose, without loss that $\int d \lambda=1$. Pick $\varepsilon^{\prime}, A>0$, so that $d \lambda\left( \pm \varepsilon^{\prime}, \pm \infty\right) \geqq A$. Let $d \lambda_{\mu}=e^{\mu \sigma} d \lambda / \int e^{\mu \sigma} d \lambda$. Then for $\mu>0$ (resp. $\left.\mu<0\right), d \lambda_{\mu}\left(\varepsilon^{\prime}, \infty\right)\left(\right.$ resp. $\left.d \lambda_{\mu}\left(-\infty,-\varepsilon^{\prime}\right)\right)$ is larger than $A$. Thus taking the contribution from $\sigma>\varepsilon^{\prime}$ (resp. $\sigma<-\varepsilon^{\prime}$ ):

$$
\begin{aligned}
P(J, \mu)-P(0, \mu) & =\lim _{|A| \rightarrow \infty}|\Lambda|^{-1} \ln \left[\int \prod_{i \in \Lambda} d \lambda_{\mu}\left(\sigma_{i}\right) \exp \left(J \sum_{|i-j|=1} \sigma_{i} \sigma_{j}\right)\right] \\
& \geqq(\ln A)+2 v J\left(\varepsilon^{\prime}\right)^{2} .
\end{aligned}
$$


Theorem 3.5. Fix a measure $d \lambda$ on $\mathbb{R}$ with $\int e^{a \sigma^{2}} d \lambda<\infty$ for all a so that supp $\mu$ is not disjoint from either $(-\infty, 0)$ or $(0, \infty)$. Then for all sufficiently large $J$, there is some $\mu_{J}$ so that $P(J, \mu)$ is not differentiable in $\mu$ at $\mu=\mu_{J}$.

Remarks. 1. This implies that $\lim _{\mu \downarrow \mu_{J}}\langle 0\rangle_{J, \mu}$ and $\lim _{\mu \uparrow \mu_{J}}\langle 0\rangle_{J, \mu}$ are distinct equilibrium states for the $\left(J, \mu_{J}\right)$ theory so there is a "phase transition".

2. By very different methods, Pirogov and Sinai [38] have proven the existence of phase transitions in systems without any symmetry.

Proof. Let $\langle 0\rangle_{J, \mu}$ be a limit of periodic states. By convexity of $P$ and the Schwartz inequality:

$$
\left\langle\sigma_{0}^{2}\right\rangle_{\sigma, \mu} \geqq \frac{1}{2 v} \sum_{ \pm, i=1}^{v}\left\langle\sigma_{0} \sigma_{ \pm \delta_{i}}\right\rangle \geqq \frac{P(J)-P(0)}{J} .
$$

Thus, by Lemma $3.42 v\left\langle\sigma_{0}^{2}\right\rangle_{\sigma, \mu} \geqq\left(\varepsilon-\delta J^{-1}\right)$. Let $c=\lim _{\alpha \rightarrow \infty}\left\langle\sigma_{\alpha} \sigma_{0}\right\rangle_{J, \mu}$.

Then, as in Theorem 3.1:

$$
2 v c(J, \mu) \geqq \varepsilon-\delta J^{-1}-v I(v) J^{-1} .
$$

Thus for all $J>J_{c}=\varepsilon / \delta+v I(v)$ and all $\mu$, there is a constant $\alpha(J)$ such that

$$
c(J, \mu) \geqq \alpha(J)>0 .
$$

We claim that (3.10) implies that for $J>J_{c}, P(J, \mu)$ is non-differentiable at some point. For suppose that it is always differentiable. Then, by a beautiful argument of Guerra [21,22] (see also Fröhlich-Simon [12]), each \langle\rangle$_{J, \mu}$ clusters so that (3.11) says:

$$
\left\langle\sigma_{0}\right\rangle_{J, \mu}^{2} \geqq \alpha(J) \text {. }
$$

Now, by mimicking the proof of Lemma 3.2

$$
\begin{aligned}
& \lim _{\mu \rightarrow \infty}\left\langle\sigma_{0}\right\rangle_{J, \mu}=\sup \{\sigma \mid \sigma \in \operatorname{supp} \lambda\}>0 \\
& \lim _{\mu \rightarrow-\infty}\left\langle\sigma_{0}\right\rangle_{J, \mu}=\inf \{\sigma \mid \sigma \in \operatorname{supp} \lambda\}<0 .
\end{aligned}
$$

Thus (3.12) implies the discontinity of $\left\langle\sigma_{0}\right\rangle_{J, \mu}$ in $\mu$ at some $\mu$ so that $P(J, \mu)$ is not differentiable at at least one point.

\section{$\S$ 4. Phase Transitions and Goldstone Bosons in $(\phi \cdot \phi)_{3}^{2}$ Quantum Field Theories}

In this section we establish phase transitions for $N$ component $(N=1,2,3) \phi^{4}$ field theories in 3 space time dimensions. In 2-dimensions and $N=1$, phase transitions have been established by Glimm, Jaffe and Spencer in [20] using a variant of Peierls' methods. One starts with a free Euclidean field $\phi=\left(\phi_{1} \ldots \phi_{N}\right)$ of mass 1 so that

$$
\left\langle\phi_{i}(x) \phi_{j}(y)\right\rangle_{0}=\delta_{i j}(2 \pi)^{-3} \int\left(p^{2}+1\right)^{-1} e^{i(x-y) \cdot p} d^{3} p .
$$


We consider the interaction in a region $\Lambda \subset \mathbb{R}^{3}$ defined by

$$
V(\Lambda)=\int_{\Lambda}\left[\lambda:(\boldsymbol{\phi} \cdot \boldsymbol{\phi})^{2}(x):-\sigma: \boldsymbol{\phi} \cdot \boldsymbol{\phi}(x):-\mu \phi_{1}(x)\right] d^{3} x+\text { c.t. }
$$

It is technically convenient to define the normal order : : in (4.1) with respect to 0 bare mass. The symbol c.t. denotes infinite counter-terms which depend only on $\lambda$ and are chosen to be $0(N)$ symmetric.

Theorem 4.1. Let $\lambda>0$ and let $\Lambda$ be the rectangle of area $|\Lambda| \geqq 1$. There is a constant $K$ such that

$$
\left\langle e^{-V(\Lambda)}\right\rangle_{0} \leqq e^{K|\Lambda|} .
$$

Moreover if $\mu \neq 0$ and $f_{i} \in \mathscr{S}\left(\mathbb{R}^{3}\right)$

$$
\lim _{\Lambda \uparrow \mathbb{R}^{3}} \frac{\left\langle\prod \phi_{j_{\imath}}\left(f_{i}\right) e^{-V(\Lambda)}\right\rangle_{0}}{\left\langle e^{-V(\Lambda)}\right\rangle_{0}} \equiv\left\langle\prod \phi_{j_{\imath}}\left(f_{i}\right)\right\rangle(\lambda, \sigma, \mu)
$$

exists and satisfies the Osterwalder-Schrader axioms including clustering.

The theorem is based on the phase space cell expansion of Glimm and Jaffe [19] who established (4.2). For small $\lambda$ and $\sigma$ Feldman-Osterwalder [7] and Magnen-Seneor [32] showed that the limit (2.3) exists and defines a quantum field model with a mass gap. Feldman-Osterwalder [8] have also established this for large $\mu$. If $N=1,2$ one can then construct a theory for all $\lambda>0, \sigma, \mu$ using correlation inequalities. See $[8,10]$. Fröhlich $[10]$ has established the theorem for $N=3$ using Lee-Yang methods provided $\mu \neq 0$. The limits $\mu \uparrow 0$ and $\mu \downarrow 0$ are denoted by $\langle 0\rangle(\lambda, \sigma, 0 \pm)$ respectively. The main result of this section is

Theorem 4.2. Fix $\sigma>1 / 2$ and $N=1,2$, or 3 . Then

$$
\lim _{\lambda \downarrow 0}\langle\phi\rangle\left(\lambda, \sigma, 0^{+}\right)=\infty
$$

which implies the presence of symmetry breaking.

Remarks. 1. The restriction on $N$ is needed only to construct Euclidean invariant states.

2. By scaling and renormal ordering we obtain (4.4) in other regions of the coupling constant space e.g. as $\sigma \uparrow \infty, \lambda$ fixed.

The proof of Theorem 4.2 follows from the following two lemmas.

Lemma 4.3. For fixed $\lambda>0, \sigma$ and $\mu$ we have

$$
\left\langle\phi_{i}(0) \phi_{i}(x)\right\rangle=c_{i}+\int_{0}^{\infty} d \varrho_{i}(a) \frac{e^{-a|x|}}{4 \pi|x|}
$$

where $\int d \varrho_{i}(a)=1$ and $\int_{0}^{\infty} a d \varrho_{i}(a)<\infty$ and $c_{i} \geqq 0$.

Moreover

$$
\left\langle: \phi_{i}^{2}(0):\right\rangle=\lim _{x \rightarrow 0}\left\{\left\langle\phi_{i}(0) \phi_{i}(x)\right\rangle-\frac{1}{4 \pi|x|}\right\}
$$

and

$$
c_{i} \geqq\left\langle: \phi_{i}^{2}(0):\right\rangle \text {. }
$$


Lemma 4.4. Fix $\sigma>1 / 2$. Then for small $\mu$

$$
\langle: \phi \cdot \phi(0):\rangle=\frac{1}{|\Omega|} \int_{\Omega} d^{3} x\langle: \phi \cdot \phi(x):\rangle \geqq b(\lambda)
$$

where $b(\lambda) \rightarrow \infty$ as $\lambda \downarrow 0$ and $\Omega$ denotes a large square.

Proof of Theorem 4.2. By a Lee-Yang argument [30, 43, 10]

$$
\lim _{x \rightarrow \infty}\left[\left\langle\phi_{i}(0) \phi_{i}(x)\right\rangle(\lambda, \sigma, \mu)-\left\langle\phi_{i}(0)\right\rangle^{2}(\lambda, \sigma, \mu)\right] \rightarrow 0
$$

for $\mu \neq 0$. Thus $c_{i}=\left\langle\phi_{i}(0)\right\rangle^{2}(\lambda, \sigma, \mu)$. By symmetry $\left\langle\phi_{i}(0)\right\rangle=0$ for $i \neq 1$. We conclude using (4.7), (4.8) and $\left\langle\phi_{1}(0)\right\rangle \geqq 0$ that

$$
\lim _{\lambda \downarrow 0}\left\langle\phi_{1}(0)\right\rangle\left\langle\lambda, \sigma, 0^{+}\right\rangle \geqq b(\lambda)^{1 / 2} \rightarrow \infty \text {. }
$$

The proof of the above lemmas relies on the following generalized $\phi$ bounds which are established in $[42,37,12]$. See $[16,22,9,20,21]$ for earlier versions.

Theorem 4.5. Let $F(\phi)$ be a function of $\phi(x)$ localized in the unit cube $\Delta_{0}$ centered at the origin. If $F(\phi)$ is invariant under reflections about each axis then

$$
\langle F(\phi)\rangle \leqq \varlimsup_{\Lambda \uparrow R^{3}} \frac{\mid\left\langle e^{-V(\Lambda)} \prod_{\Delta \subset \Delta} F_{\Delta}(\phi)\right\rangle_{0}^{1 /|\Lambda|}}{\left\langle e^{-V(\Lambda)}\right\rangle_{0}^{1 /|\Lambda|}} .
$$

The above product ranges over lattice squares and $F_{\Delta}$ denotes the translate of $F$ localized in $\Delta$.

Proof of Lemma 4.3. Formula (4.5) is the analytic continuation of the KällenLehmann representation $[27,31,39]$ which is valid for Wightman field theories. The conditions on the moments of $d \varrho$ follow from (4.6). To see this note that by (4.6) and the finiteness of $\left\langle: \phi_{i}^{2}:\right\rangle$

$$
\lim _{x \rightarrow 0}|x|\left\langle\phi_{i}(0) \phi_{i}(x)\right\rangle=(4 \pi)^{-1} .
$$

By (4.5) and the monotone convergence theorem the above limit equals $(4 \pi)^{-1} \int d \varrho_{i}(a)$ so $\int d \varrho_{i}(a)=1$. From (4.5) and (4.6) we have

$$
c_{i}=\left\langle: \phi_{i}^{2}(0):\right\rangle+(4 \pi)^{-1} \int_{0}^{\infty} a d \varrho_{i}(a)
$$

from which (4.7) follows. Thus we need only prove (4.6) which concerns the interchange of volume and momentum cutoff limits. We apply Theorem 4.5 with

$$
F=\int_{\left|x_{j}\right| \leqq 1 / 3}\left[: \phi_{i}^{2}(x):-\phi_{i}\left(x-\frac{\delta}{2}\right) \phi_{i}\left(x+\frac{\delta}{2}\right)-(4 \pi \delta)^{-1}\right] d^{3} x .
$$

As $\delta \downarrow 0$ the phase space cell expansion shows

$$
\lim _{\delta \downarrow 0}\left|\left\langle\prod_{\Delta \subset A} F_{\Delta} e^{-V(\Lambda)}\right\rangle_{0}\right| 1 /|\Lambda|=0
$$

uniformly in $\Lambda$ because each Feynman graph arising from $F$ tends to 0 . 
Proof of Lemma 4.4. Let $\Omega$ be a large cube and define

$$
\begin{aligned}
: \phi^{2}:(\Omega) & =\sum_{i=1}^{N} \int_{\Omega}: \phi_{i}^{2}(x): d x \\
\exp \alpha_{\infty}(\lambda, \sigma, \mu) & =\lim _{\Lambda \uparrow R^{3}}\left\langle e^{-V(\Lambda)}\right\rangle^{1 /|\Lambda|}(\lambda, \sigma, \mu) .
\end{aligned}
$$

See $[42,21,37]$ for the convergence of (4.9). By Theorem 4.5, generalized from unit cubes to large cubes, we have

$$
\begin{aligned}
& \left\langle e^{-\sigma: \phi:^{2}(\Omega)}\right\rangle(\lambda, \sigma, \mu)\left\langle e^{+\sigma: \phi:^{2}(\Omega)}\right\rangle(\lambda, 0, \mu) \\
& \leqq \exp \left[\alpha_{\infty}(\lambda, 0, \mu)-\alpha_{\infty}(\lambda, \sigma, \mu)+\alpha_{\infty}(\lambda, \sigma, \mu)-\alpha_{\infty}(\lambda, 0, \mu)\right]|\Omega|=1 .
\end{aligned}
$$

By Jensen's inequality and (4.10)

$$
e^{-\sigma\left\langle\phi::^{2}(\Omega)\right\rangle(\lambda, \sigma, \mu)} \leqq\left\langle e^{-\sigma: \phi:^{2}(\Omega)}\right\rangle(\lambda, \sigma, \mu) \leqq\left[\left\langle e^{+\sigma: \phi:^{2}(\Omega)}\right\rangle(\lambda, 0, \mu)\right]^{-1} .
$$

Thus it suffices to prove that $\left\langle e^{+\sigma: \phi:^{2}(\Omega)}\right\rangle(\lambda, 0, \mu)$ tends to $\infty$ as $\lambda \downarrow 0$. By Theorem 4.5 , $\left\langle\exp \left(-\sigma: \phi^{2}:(\Omega)\right)\right\rangle(\lambda, 0, \mu)$ is bounded by $\exp \left[|\Omega|\left(\alpha_{\infty}(\lambda,-\sigma, \mu)-\alpha_{\infty}(\lambda, 0, \mu)\right)\right]$ which is uniformly bounded for small $\lambda>0$ and $|\mu| \leqq \mu_{0}$ (see $[7,21,42]$ ). Hence we need only show that

$$
\begin{aligned}
& \lim _{\lambda \downarrow 0}\left\langle\cosh \left(\sigma: \phi^{2}:(\Omega)\right)\right\rangle(\lambda, 0, \mu) \\
& =\lim _{\lambda \downarrow 0} \sum_{M=0}^{\infty}[(2 M) !]^{-1}\left\langle\left[\sigma: \phi:^{2}(\Omega)\right]^{2 M}\right\rangle(\lambda, 0, \mu)=\infty .
\end{aligned}
$$

Since each term of the above sum is positive and asymptotic for small $\lambda$, we have

$$
\lim _{\lambda \downarrow 0}\left\langle\cosh \sigma: \phi^{2}:(\Omega)\right\rangle(\lambda, 0, \mu) \geqq\left\langle\cosh \sigma: \phi^{2}:(\Omega)\right\rangle(0,0, \mu) .
$$

The last expectation is the free field expectation and is infinite for $\sigma>1 / 2$ and large $|\Omega|$ by a direct computation. For example, one can get a lower bound by conditioning [23] if we put Dirichlet boundary conditions on $\partial \Omega$ where a normal mode expansion suffices.

The proof of Theorem 4.2 holds whenever the infinite volume field theory is Euclidean invariant. For $N>3$ the Lee-Yang theorem is not known but we can construct the infinite volume limit by considering expectations in a periodic box $\Lambda$ and taking subsequences. Here we use the $\phi$ bounds for periodic boundary conditions to obtain compactness of $\langle 0\rangle_{A}$. The limiting theory may not be Euclidean invariant - so that the Källen-Lehmann representation does not hold. Nevertheless the grad $\phi$ bounds (2.3) apply by taking the lattice spacing to zero and using Park's theorem on the convergence of the lattice approximation [36]. This yields

$$
F(x) \equiv\langle\phi(x) \phi(0)\rangle=c+\int e^{i k \cdot x} f(k) d^{3} k /(2 \pi)^{3}
$$

where $f(k) \leqq k^{-2}$. It follows that $F(x)-c-(4 \pi)^{-1}|x|^{-1}$ is negative definite so that

$$
c \geqq \lim _{x \rightarrow 0}\left[\langle\phi(x) \phi(0)\rangle-(4 \pi)^{-1}|x|^{-1}\right] .
$$

This replaces Lemma 4.3. Lemma 4.4 holds uniformly in $\Lambda$. The proof now follows as before. 
Remark. As an important consequence of Theorem 4.2 we mention that combining it with some estimates on the renormalized, locally conserved current (which should be checked), one obtains the existence of $N-1 \quad(N=2,3)$ Goldstone bosons ( $=0$ mass one particle states) in the regime where $\left\langle\phi_{1}\right\rangle_{+} \neq 0$. This is a general axiomatic result of $[6,45]$.

\section{Appendix. Alternate Proof of Theorem 2.3}

In this appendix we give an alternate proof of Theorem 2.3 (with an extra factor of $2 v$ ). This proof is motivated by a proof of the Kallen-Lehmann representation (1.2).

Fix a direction, say the one labeled by $\alpha_{1}$. Let $\alpha=\left(\alpha_{1}, \alpha^{\prime}\right)$ and let $g\left(\alpha^{\prime}\right) \in R^{N}$ be a function of the lattice sites in a hyperplane. We define an averaged spin variable

$$
\sigma_{j}(g)=\sum_{\alpha^{\prime}} g\left(\alpha^{\prime}\right) \cdot \sigma_{\left(j, \alpha^{\prime}\right)}
$$

Let $T$ be the transfer matrix in the 1 direction defined by

$$
T=G T_{0} G
$$

where $T_{0}$ is given in $\S 2$ and $G=F^{1 / 2}$ in (2.5) with $h=0$. Let $s(g)$ be the operator on $L^{2}\left(R^{M}\right)$ given by multiplication by $\sigma_{0}(g)$.

Theorem A.1. Let $\langle 0\rangle$ be the finite volume expectation. Then

$$
\begin{aligned}
0 & \leqq 2\left(1-\cos k_{1}\right) \sum_{j=0}^{L_{1}-1}\left\langle\sigma_{0}(\bar{g}) \sigma_{j}(g)\right\rangle e^{i k_{1} \cdot j} \\
& \leqq 2 \operatorname{tr}\left\{[s(\bar{g}),[s(g), T]] T^{L_{1}-1}\right\} \\
& \leqq \frac{2\|g\|^{2}}{J} \equiv \frac{2}{J} \sum_{\alpha^{\prime}}\left|g\left(\alpha^{\prime}\right)\right|^{2} .
\end{aligned}
$$

If we set $g\left(\alpha^{\prime}\right)=e^{i p^{\prime} \cdot \alpha^{\prime}}$ then by (A.1) we obtain

$$
2\left(1-\cos k_{1}\right) F(k) \leqq 2 J^{-1}
$$

where $F(k)$ is the Fourier transform of the two point function. Since the one direction is arbitrary we can sum over all $v$ directions to obtain Theorem 2.3 with an extra factor of $2 v$ on the right.

The proof of (A.1) relies on two lemmas.

Lemma A.2. If $0<r<1$ and $k_{1}$ is a multiple of $2 \pi / L_{1}$ then

$$
\begin{aligned}
& 2\left(1-\cos k_{1}\right) \sum_{\alpha_{1}=0}^{L_{1}-1}\left(r^{\alpha_{1}}+r^{L_{1}-\alpha_{1}}\right) e^{i \alpha_{1} k_{1}} \\
& =2\left(1-\cos k_{1}\right)\left(1-r^{L_{1}}\right)\left(1-r^{2}\right)\left|1-e^{i k_{1}} r\right|^{-2} \\
& \leqq 4\left[\left(1+r^{L_{1}}\right)-\left(r+r^{L_{1}-1}\right)\right] .
\end{aligned}
$$


The proof is elementary using the identity $\sum_{m=0}^{L-1} x^{m}=\left(1-x^{L}\right) / 1-x$ and the inequalities

$$
2\left(1-\cos k_{1}\right) \leqq 4\left|1-e^{i k_{1}} r\right|^{2}(1+r)^{-2}
$$

and $\left(1-r^{L_{1}}\right) \leqq(1+r)\left(1-r^{L_{1}-1}\right)$ for $0<r<1$.

Lemma A.3. Let $T$ and $s(g)$ be defined as above. Then

$$
\frac{1}{2}[s(\bar{g}),[s(g), T]] \leqq(2 J)^{-1}\|g\|^{2} T .
$$

Proof. First note that since $s$ is a multiplication operator and commutes with $G$ it suffices to establish (A.3) with $T$ replaced by $T_{0}$. In momentum space, $T_{0}$ is multiplication by

$e^{-\sum_{\alpha^{\prime}} k_{\alpha^{\prime}}^{2} / 2 J}$

and $s(g)$ is the derivative $-i \sum g\left(\alpha^{\prime}\right) \frac{\partial}{\partial k_{\alpha^{\prime}}}$. In this representation the commutator (A.3) is

$$
\begin{aligned}
& \sum_{\alpha^{\prime}, \beta^{\prime}} \bar{g}\left(\alpha^{\prime}\right) g\left(\beta^{\prime}\right) \frac{\partial^{2}}{\partial k\left(\alpha^{\prime}\right) d k\left(\beta^{\prime}\right)} e^{-\sum k_{\alpha}^{2} / 2 J} \\
& =\left[\frac{\|g\|^{2}}{J}-\frac{\left|\sum_{\alpha^{\prime}} g\left(\alpha^{\prime}\right) \cdot k_{\alpha^{\prime}}\right|^{2}}{J^{2}}\right] e_{\alpha^{\prime}} k_{\alpha}^{2} / 2 J \\
& \leqq 2 J^{-1}\|g\|^{2} T_{0} .
\end{aligned}
$$

Proof of Theorem A.1. Let $e_{i}$ be the eigenvectors for $T$ with eigenvalues $\lambda_{i}$ ordered so that $\lambda_{1} \geqq \lambda_{2} \geqq \lambda_{m}$. Define the symmetric matrix

$$
c_{m n}=\left(e_{m}, s(g) e_{n}\right)_{L^{2}} .
$$

Then we have

$$
\begin{aligned}
& \sum_{j=0}^{L_{1}-1}\left\langle\sigma_{0}(\vec{g}) \sigma_{j}(g)\right\rangle e^{i j \cdot k_{1}} \equiv F_{g}\left(k_{1}\right) \\
& =\sum_{j=0}^{L_{1}-1} \operatorname{tr}\left[s(\vec{g}) T^{j} S(g) T^{L_{1}-j}\right] e^{i j \cdot k_{1}} \\
& =\sum_{j=0}^{L_{1}-1} \sum_{m, n} \bar{c}_{m n} c_{n m} \lambda_{m}^{j} \lambda_{n}^{L_{1}-j} e^{i j \cdot k_{1}} \\
& =\sum_{j=0}^{L_{1}-1} \sum_{m<n}\left|c_{m n}\right|^{2} \lambda_{m}^{L_{1}}\left[\left(\frac{\lambda_{n}}{\lambda_{m}}\right)^{j}+\left|\frac{\lambda_{n}}{\lambda_{m}}\right|^{L_{1}-j}\right] e^{i j k_{1}} .
\end{aligned}
$$

Now we apply Lemma A.2 with $r=\lambda_{n} \lambda_{m}^{-1}$ and we obtain

$$
\begin{aligned}
2\left(1-\cos k_{1}\right) F_{g}\left(k_{1}\right) & \leqq 4 \sum_{m<n}\left|c_{m n}\right|^{2} \lambda_{m}^{L_{1}}\left(1+r^{L_{1}}-r-r^{L_{1}-1}\right) \\
& =4 \operatorname{tr}\left[s(\vec{g}) s(g) T^{L_{1}}-s(\vec{g}) T s(g) T^{L_{1}-1}\right] \\
& =2 \operatorname{tr}\left\{[s(\bar{g}),[s(g), T]] T^{L_{1}-1}\right] \\
& \leqq 2 J^{-1}\|g\|^{2}
\end{aligned}
$$


where Lemma A.3 was applied in the last inequality and this completes the proof.

\section{References}

1. Beijeren,H. van, Sylvester, G.: Phase transitions for continuous spin ising ferromagnets, Yeshiva preprint

1A. Bortz,A., Griffiths,R.: Phase transitions in anisotropic classical Heisenberg ferromagnets. Commun. math. Phys. 26, 102-108 (1972)

2. Coleman, S.: There are no Goldstone bosons in two dimensions. Commun. math. Phys. 31, 259-264 (1973)

3. Dobrushin,R.L., Shlosman,S.B.: Absence of breakdown of continuous symmetry in twodimensional models of statistical physics. Commun. math. Phys. 42, 31 -40 (1975)

4. Domb,C., Green, M.: Phase transitions and critical phenomena, Vol. 3, Series expansions for lattice models. New York-London: Academic Press 1974

5. Dunlop,F., Newman, C.: Multicomponent field theories and classical rotators. Commun. math. Phys. 44, 223-235 (1975)

6. Ezawa, H., Swieca, A.: Spontaneous breakdown of symmetry and zero mass states. Commun. math. Phys. 5, 330-336 (1967)

7. Feldman, J., Osterwalder, K.: The Wightman axioms and the mass gap for weakly coupled $\left(\phi^{4}\right)_{3}$ quantum field theories. Ann. Phys., to appear

8. Feldman, J., Osterwalder, K.: in preparation

9. Fröhlich, J.: Schwinger functions and their generating functionals. I. Helv. Phys. Acta 74, 265-306 (1974)

10. Fröhlich, J.: Existence and analyticity in the bare parameters of the $\lambda(\phi \cdot \phi)^{2}-\sigma \phi_{1}^{2}-\mu \phi_{1}$ quantum field models; see Proc. Marseille Conf.

11. Fröhlich, J.: in preparation, see also 1976 Schladming Lecture Notes

12. Fröhlich,J., Simon, B.: Pure states for general $P(\phi)_{2}$ Theories: Construction, regularity and variational equality, Princeton Preprint

13. Fröhlich, J., Simon, B., Spencer, T.: Phase transitions and continuous symmetry breaking. Phys. Rev. Letters 36, 804 (1976)

14. Gallavotti, G., Miracle-Sole, S.: Equilibrium states of the Ising model in the two phase region. Phys. Rev. 5B, 2555 (1972)

15. Ginibre, J.: Existence of phase transitions for quantum lattice systems. Commun. math. Phys. 14, $205-234(1969)$

16. Glimm,J., Jaffe, A.: The $\lambda\left(\phi^{4}\right)_{2}$ quantum field theory without cutoffs. IV. Perturbations of the Hamiltonian. J. Math. Phys. 13, 1568-1584 (1972)

17. Glimm,J., Jaffe, A.: Energy-momentum spectrum and vacuum expectation values in quantum field theory. I. J. Math. Phys. 11, 3335-3338 (1970)

18. Glimm, J., Jaffe, A.: $\phi_{2}^{4}$ quantum field model in the single phase region: Differentiability of the mass and bounds on critical exponents. Phys. Rev. D10, 536-539 (1974)

19. Glimm, J., Jaffe, A.: Positivity of the $\left(\phi^{4}\right)_{3}$ Hamiltonian. Fortschr. Physik 21, $327-376$ (1973)

20. Glimm, J., Jaffe, A., Spencer, T.: Phase transitions for $\phi_{2}^{4}$ quantum fields. Commun. math. Phys. 45, 203-216 (1975)

20A. Griffiths, R.: Phase transitions. In: Statistical mechanics and quantum field theory (ed. De Witt and Stora). New York-London: Gordon and Breach 1970

21. Guerra, F.: Proc. Marseille Conf. 1976 and Proc. Bielefeld Symposium, 1976

22. Guerra,F., Rosen,L., Simon, B.: Nelson's symmetry and the infinite volume behavior of the vacuum in $P(\phi)_{2}$. Commun. math. Phys. 27, 10-22 (1972)

23. Guerra,F., Rosen, L., Simon, B.: The $P(\phi)_{2}$ euclidean quantum field theory as classical statistical mechanics. Ann. Math. 101, 111--259 (1975)

24. Herbst, I.: Some remarks on canonical quantum field theories. Princeton Preprint, 1976

25. Hohenberg, P.: Existence of long-range order in one and two dimensions. Phys. Rev. 158, 383 (1967)

26. Kac, M.: On applying mathematics: Reflections and examples. Quart. Appl. Math. 30, 17-29 (1972) 
27. Källen, G.: On the definitions of the renormalization constants in quantum electrodynamics. Helv. Phys. Acta 25, 417-434 (1952)

28. Katsura, S., Inawashiro, S., Abe,Y.: Lattice Green's functions for the simple cubic lattice in terms of a Mellin-Barnes type integral. J. Math. Phys. 12, 895-899 (1971)

29. Kunz,H., Pfister, Ch.-Ed., Vuillermot, P. A.: Inequalities for some classical spin vector models. Bielefeld Preprint

30. Lee,T.D., Yang, C.N.: Statistical theory of equations of state and phase transitions. II. Lattice Gas and Ising Model. Phys. Rev. 87, 410-419 (1952)

31. Lehmann, H.: Über Eigenschaften von Ausbreitungsfunktionen and Renormierungskonstanten quantisierter Felder. Nuovo Cimento 11, 417-434 (1954)

32. Magnen, J., Seneor, R.: The infinite volume limit of the $\phi_{3}^{4}$ model. Ann. Inst. Henri Poincaré, to appear

33. Malyshev, S.: Phase transitions in classical Heisenberg ferromagnets with arbitrary parameter of anisotropy. Commun. math. Phys. 40, 75-82 (1975)

34. Mermin, N.D.: Absence of ordering in certain classical systems. J. Math. Phys. 8, 1061-1064 (1967)

35. Mermin, N.D., Wagner, H.: Absence of ferromagnetism or antiferromagnetism in one or two dimensional isotropic Heisenberg models. Phys. Rev. Letters 17, 1133 (1966)

36. Park, Y.: Lattice approximation of the $\left(\lambda \phi^{4}-\mu \phi\right)_{3}$ field theory in a finite volume. J. Math. Phys.16, 1065 (1975)

37. Park, Y.: Uniform bounds on the pressure of the $\lambda \phi_{3}^{4}$ model, and uniform bounds on the Schwinger functions in Boson field models. Bielefeld Preprints

38. Pirogov, S.A., Sinai, Ya.G.: Phase transitions of the first kind for small perturbations of the ising model. Funct. Anal. Appl. 8, 21-25 (1974)

39. Reed, M., Simon, B.: Methods of modern mathematical physics, Vol. II, Fourier Analysis, SelfAdjointness. New York-London: Academic Press 1975

40. Robinson, D.: A proof of the existence of phase transitions in the anisotropic Heisenberg model. Commun. math. Phys. 14, 195 (1969)

41. Ruelle, D.: Statistical mechanics. New York: Benjamin 1969

42. Seiler, E., Simon, B.: Nelson's symmetry and all that in the Yukawa 2 and $\phi_{3}^{4}$ field theories. Ann. Phys. to appear

43. Simon, B.: Correlation inequalities and the mass gap in $P(\phi)_{2}$. II. Uniqueness of the vacuum in a class of strongly coupled theories. Ann. Math. 101, 260-267 (1975)

44. Stanley, H.: Phys. Rev. 176, 718 (1968)

45. Symanzik, K.: Commun. math. Phys. 6, 228 (1967)

46. Watson, G. N.: Three triple integrals. Quart. J. Math. 10, 266 (1939)

Communicated by J. L. Lebowitz

Received May 12, 1976 
